\title{
Response of citrus hybrids to Alternaria alternata inoculation
}

\author{
Citriculture Center 'Sylvio Moreira' Agronomic Institute - IAC, Cordeirópolis, Brazil \\ *Corresponding author, e-mail: gabi.026@hotmail.com
}

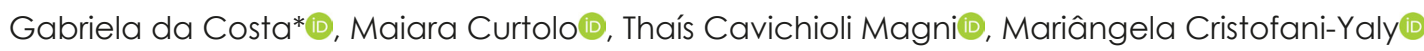

\begin{abstract}
Citrus orchards have some limitations, such as the occurrence of phytosanitary problems. Alternaria brown spot (ABS) is caused by fungus Alternaria alternata, which affects several parts of the plant by producing a host-specific toxin, known as ACT. ABS is a limiting factor in orchards due to the susceptibility of most planted cultivars: 'Murcott' tangor and 'Ponkan' tangerine. The selection of varieties resistant/tolerant to the disease has economic importance. Therefore, the aim of this experiment was to evaluate the response to A. alternata inoculation in a population of 'Murcott' tangor vs 'Pera' sweet orange hybrids. Leaves of 2-3 centimeters in length of 'Murcott' tangor, 'Pera' sweet orange, 'Ponkan', 'Dancy', 'Fremont' tangerine and 198 hybrids were collected. For in vitro inoculation, monosporic A. alternata culture at concentration of $10^{5} \mathrm{conidia} \mathrm{mL}^{-1}$ was used. Inoculated leaves were stored in humid chamber. After 24, 48 and 72 hours of inoculation, leaf lesions were evaluated following a diagrammatic scale. The results obtained showed that most hybrids from the crossing of 'Murcott' tangor vs 'Pera' sweet orange are susceptible to ABS. However, 44 are resistant and ten are tolerant. Among ABS-tolerant hybrids, some have phenotype similar to that of cultivated and commercialized hybrids.
\end{abstract}

Keywords: Citrus, phytopathology, alternaria brown spot, mandarin, tangor

\section{Introduction}

The third predominant group in Brazilian citrus orchards is composed of tangerines and their hybrids, which are produced for the fresh fruit market (IBGE, 2018). Varieties that stand out are 'Ponkan' tangerine (Citrus reticulata Blanco) and 'Murcott' tangor hybrid (Citrus reticulata x Citrus sinensis (L.) Osbeck) (Bastianel et al., 2014).

Despite being competitive, Brazilian citrus has limitations, reflected in its planted area and production, which have suffered falls in recent years (Barros et al., 2016), which is the result of the reduced number of varieties and the monoculture system adopted, that is, by the narrow genetic base. These factors made the crop vulnerable to phytosanitary problems, which result in low productivity (Sousa \& Goes, 2010; Wu et al., 2014). One of the main diseases that limits production and cultivation of tangerines is the alternaria brown spot (ABS).
ABS is caused by filamentous fungus $A$. alternata f. sp. citri (Azevedo et al., 2019). The disease was first observed in Brazil in 2001 in the 'Dancy' tangerine variety (Citrus reticulata Blanco) (Goes et al., 2001). Symptoms usually appear 24 to 48 hours after infection, affecting immature green tissues, causing defoliation and dryness in branches. In fruits, it promotes fall and the appearance of depressed lesions. Symptoms are characterized by black or brown spots, surrounded or not by yellow halos (Azevedo et al., 2015). Necrotic areas are the result of the action of the host-specific toxin, in the case of the tangerine pathotype, the toxin is ACT (Alternaria Citri Tangerine) (Tsuge et al., 2013). The fungus is saprophytic, surviving in plants remains and its reproduction is characterized by the production of conidia (Azevedo et al., 2010).

According to Bastianel et al. (2014), commercial varieties, such as 'Dancy' and 'Ponkan' tangerines 
and 'Murcott' tangor are susceptible to ABS. Due to susceptibility, there is reduction in harvested area and increase in production costs, since orchards with susceptible varieties require more than 10 sprays per year to control the disease (Soriano et al., 2012). However, there are also resistant species, such as sweet oranges, 'Tahiti' acid lime (Citrus latifolia Tanaka), 'Montenegrina' tangerine (Citrus delicious Tenore) and 'Fremont' tangerine (Citrus reticulata Blanco x Citrus clementina Hort. Ex Tanaka) (Soriano et al., 2012; Bastianel et al., 2014).

Genetic improvement should be highlighted in the selection of resistant varieties, as it enables the introduction of superior genotypes, adapted to edaphoclimatic conditions and resistant to diseases. Hybridization is one of the most used tools in breeding programs (Spósito et al., 2003). Varieties that are not infected by the fungus can be used as source of resistance in crosses directed to obtain ABS-resistant hybrids.

It is understood that ABS represents losses for the sector, mainly due to the susceptibility of the main tangerine varieties. Thus, the aim of the present study was to evaluate the response of hybrids from the crossing of 'Murcott' tangor vs 'Pera' sweet orange (Citrus sinensis) to the in vitro inoculation of fungus A. alternata f. sp. citri, aiming to find tolerant or resistant varieties and attractive to the consumer market.

\section{Material and Methods}

Plant material

Hybrids were obtained in 1997 after controlled crosses between 'Murcott' tangor and 'Pera' sweet orange from the Citrus Germplasm Bank, at the "Sylvio Moreira" Citriculture Center of "Instituto Agronômico" IAC, located in Cordeirópolis-SP. Zygotic embryos were identified by using SSR markers (Oliveira et al., 2002) and grafted on 'Rangpur' lime (Citrus limonia Osbeck).

A total of 335 hybrids were obtained, which were established in 2015 in greenhouse; however, only 198 hybrids were included in the experiment. The two respective parents and three controls, 'Ponkan' and 'Dancy' tangerines, both susceptible to ABS and ABSresistant 'Fremont' tangerine (Azevedo et al., 2010) were also evaluated in the presence of $A$. alternata isolate. The experiment was established at the "Sylvio Moreira" - IAC Citriculture Center.

\section{Inoculation isolation and preparation}

A. alternata isolate was obtained from 'Murcott' tangor fruit tissues with typical lesions, collected from plants maintained in field at the "Sylvio Moreira" - IAC
Citriculture Center, where the fungus occurs endemically. Injured tissues were cut, selected and superficially disinfected with immersion in $70 \%$ ethanol, $3 \%$ sodium hypochlorite and autoclaved distilled water.

Tissues were incubated in Petri dishes containing BDA culture medium (200 g potato, $20 \mathrm{~g}$ dextrose and 15 $g$ agar $\mathrm{L}^{-1}$ ) and carbendazin fungicide at concentration of $640 \mathrm{mg} \mathrm{L}^{-1}$ of i.a. to inhibit the growth of opportunistic fungi and because A. alternata has high degradation capacity of this fungicide (Pacheco et al., 2012; Huang et al., 2015). Petri dishes were kept in BOD incubator with 12 -hour photoperiod at $27^{\circ} \mathrm{C}$. A monosporic culture was obtained from the initial isolate, according to adaptation of the methodology described by Silva et al. (2009).

After incubation period of approximately five days, the isolate was identified by means of asexual reproduction structures, using optical microscopy, observing dark colored conidia, with cross-sectional and longitudinal septa (Figure 1). The pathogenicity of the isolate was validated under laboratory conditions with in vitro inoculation tests on leaves of species known to respond to A. alternata such as 'Pera' sweet orange and 'Fremont' and 'Dancy' tangerines. After validating the pathogenicity, the experiment was continued with the other Petri dishes to prepare the inoculum solution.

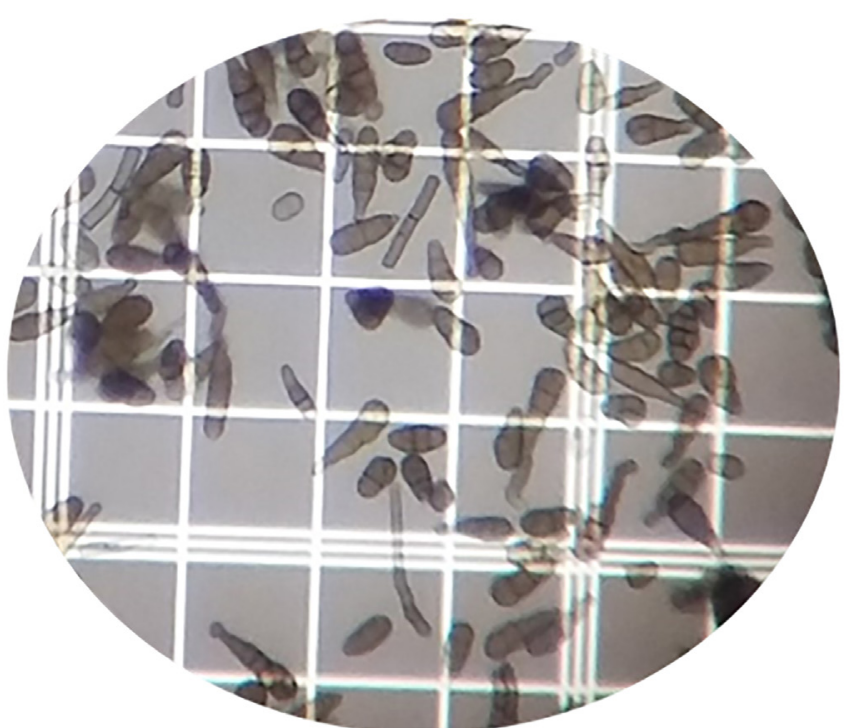

Figure 1. A. alternata f. sp. citri conidia and hyphae observed under microscope (40x).

The conidia suspension for leaf inoculation was obtained by adding $10 \mathrm{~mL}$ of autoclaved distilled water to the surfaces of Petri dishes. Conidia were removed with the aid of a sterile spatula, thus, obtaining the suspension, which was filtered with a double layer of sterile gauze to remove mycelial fragments. The final concentration was adjusted using hemocytometer (Neubaver chamber) for 
$10^{5}$ conidia $\mathrm{mL}^{-1}$.

In vitro Inoculation and evaluation of symptoms

For in vitro inoculations, leaves of branches in the V4 stage of vegetative development were used, standardizing with two to three centimeters in length. Petri dishes were lined with filter paper and a portion of cotton wool moistened with autoclaved distilled water to form a moist chamber and ensure favorable conditions for fungus infection. Leaves were packed with the abaxial side facing upwards (four leaves per plate) and $2.0 \mathrm{~mL}$ of conidia suspension were sprayed, according to methodology described by Peever et al. (1999), Canihos et al. (1999) and Turgutoğlu \& Baktir (2019). Petri dishes were kept in BOD incubator chamber at temperature of $27^{\circ} \mathrm{C}$ and 12-hour photoperiod. Disease evaluations were performed 24, 48 and 72 hours after inoculation, using the diagrammatic scale described by Martelli et al. (2016). The scale represents the level of symptoms in ten scores, in which zero represents leaf without symptoms, and scores from one to nine represent, respectively, $0.3 ; 3.5$; 8.0; $15.0 ; 34.0 ; 61.0 ; 80.0 ; 90.0$ and $97.0 \%$ of leaf area with A. alternata symptoms.

The area under the disease progress curve (AUDPC) was calculated using the formula: AUDPC = $\Sigma[(y 1+y 2) / 2 *(\dagger 2-\dagger 1)]$, in which $y=$ severity and $t=$ time unit (Shaner \& Finey, 1977). The experimental design was completely randomized, containing one treatment with three times (24, 48 and 72 hours), its control and four replicates. For statistical analysis, the SASM - Agri software (Canteri et al., 2001) was used. Data were transformed into square root $(Y+1)$ and tests of analysis of variance and comparison of means were performed using the Scott-Knott test at $5 \%$ probability level.

\section{Results and discussion}

Symptoms were observed 24 hours after fungus inoculation on leaves, such as small brown to black spots of varying size, characteristic ABS symptoms, which, in some cases, have expanded to large necrotic areas of leaf tissues due to the action of the specific-fungus toxin (ACT) (Akimitsu et al., 2014). In susceptible hybrids, such as TMXLP 241 and TMXLP 34, lesions gradually increased with time and the most severe lesions were found at time of 72 hours (Figure 2).

As expected, 'Ponkan' and 'Dancy' tangerines and 'Murcott' tangor presented lesions characteristic of alternaria brown spot, confirming their susceptibility (Bastianel et al., 2014) and differing statistically from 'Fremont' tangerine and 'Pera' sweet orange, used as controls (Porcino et al., 2017) resistant to the isolate that causes ABS (Figure 3). The resistance of 'Pera' sweet orange is explained by the fact that the tangerine pathotype of A. alternata f. citri sp affect only tangerines and their respective hybrids, since it produces hostspecific toxins (Akimitsu et al., 2014).

In the hybrid population, great variability was observed in response to A. alternata inoculation (Table 1).

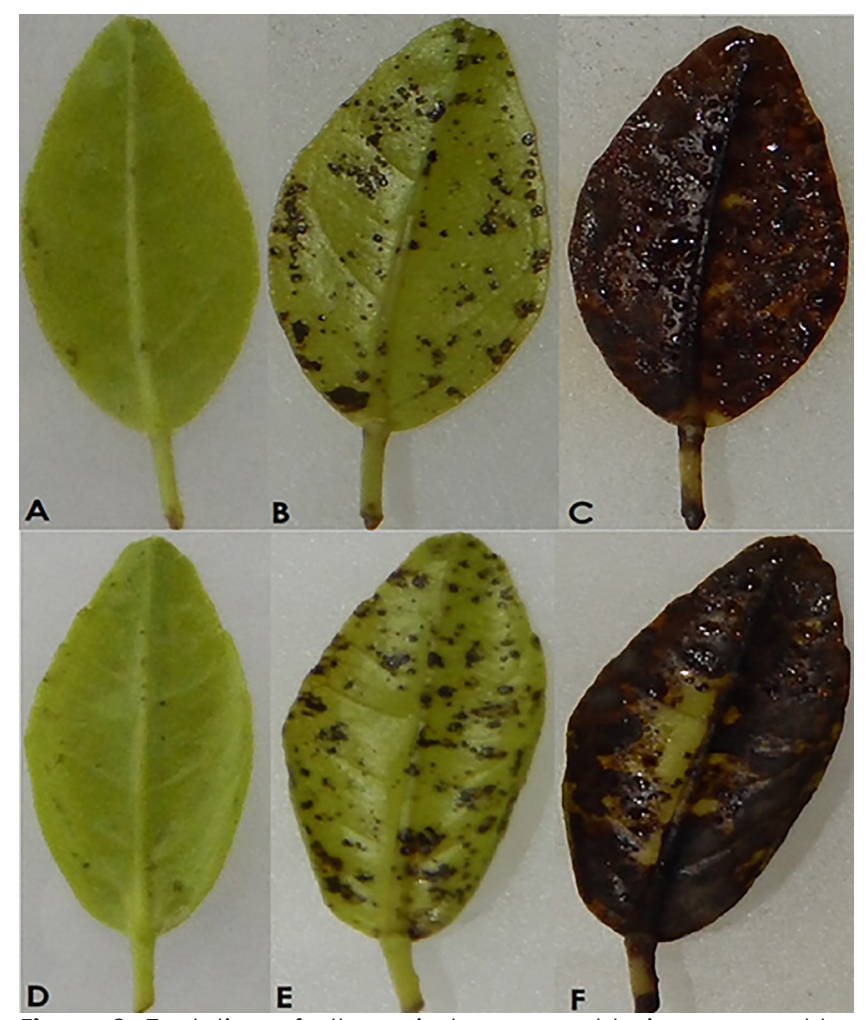

Figure 2. Evolution of alternaria brown spot lesions caused by Alternaria alternata in TMxLP 241 (A - 24 hours, B - 48 hours, C - 72 hours) and TMxLP 34 hybrids (D - 24 hours, E - 48 hours, F - 72 hours).

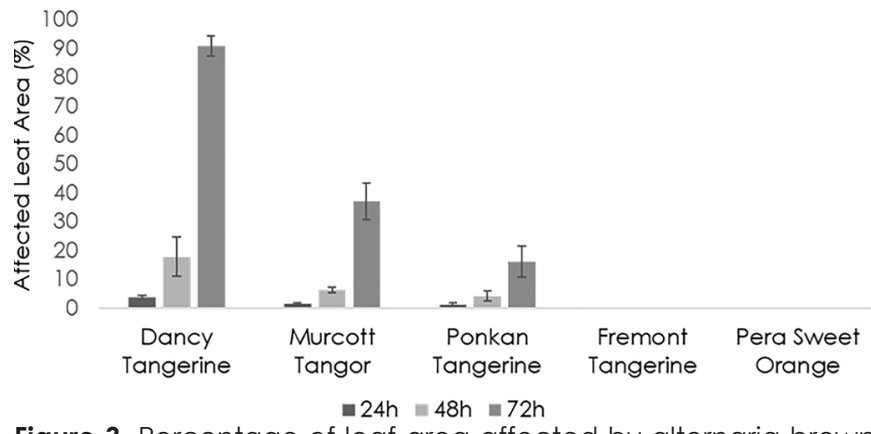

Figure 3. Percentage of leaf area affected by alternaria brown spot in varieties used as controls after 24,48 and 72 hours of inoculation.

After 24 hours, 142 hybrids and three controls showed symptoms ranging from 0 to $15 \%$ of affected leaf area, corresponding to $71.43 \%$ of the population. In the 48-hour evaluation, eight more hybrids showed symptoms, ranging from 0 to $61 \%$ of affected leaf area, totaling 153 symptomatic individuals, corresponding 
Table 1. Severity (\%) after 24,48 and 72 hours of inoculation and area under the disease progress curve (AUDPC), caused by Alternaria alternata in 'Murcott' tangor vs. 'Pera' sweet orange hybrids, their respective parents and controls, negative ('Fremont' tangerine) and positive ('Ponkan' tangerine) (Cordeirópolis, 2018).

\begin{tabular}{|c|c|c|c|c|c|c|c|c|}
\hline \multicolumn{8}{|c|}{ Symptoms after inoculation (\%) } & \\
\hline Individuals & $24 \mathrm{~h}$ & & $48 \mathrm{~h}$ & & $72 \mathrm{~h}$ & & AUDPC & \\
\hline TMXLP 72 & 2.30 & $d$ & 12.69 & C & 97.00 & a & 1496.16 & b \\
\hline TMxLP 234 & 0.66 & e & 10.94 & d & 97.00 & a & 1434.48 & b \\
\hline TMXLP 361 & 4.06 & c & 54.38 & $a$ & 97.00 & a & 2517.84 & a \\
\hline TMxLP 7 & 2.30 & $d$ & 15.00 & $c$ & 96.13 & a & 1541.16 & b \\
\hline TMxLP 305 & 0.70 & e & 15.63 & C & 96.13 & a & 1537.08 & b \\
\hline TMXLP 286 & 1.50 & $d$ & 8.00 & d & 96.13 & a & 1363.56 & b \\
\hline TMxLP 124 & 1.90 & $d$ & 13.25 & $c$ & 94.88 & a & 1479.36 & b \\
\hline TMxLP 244 & 3.26 & c & 36.00 & $a$ & 94.88 & a & 2041.68 & a \\
\hline TMXLP 100 & 0.63 & e & 11.25 & d & 92.75 & a & 1390.56 & b \\
\hline 'Dancy' & 3.70 & c & 17.90 & $c$ & 91.00 & a & 1566.00 & b \\
\hline TMxLP 208 & 0.70 & e & 44.63 & $a$ & 90.38 & a & 2164.08 & a \\
\hline TMxLP 225 & 8.06 & $b$ & 44.12 & $a$ & 90.12 & a & 2237.04 & a \\
\hline TMxLP 203 & 3.10 & c & 14.13 & $c$ & 87.38 & a & 1424.88 & b \\
\hline TMxLP 192 & 2.30 & $d$ & 11.41 & $d$ & 86.75 & a & 1342.44 & $b$ \\
\hline TMxLP 61 & 0.86 & e & 5.63 & e & 86.73 & a & 1186.20 & $b$ \\
\hline TMxLP 80 & 2.82 & c & 27.88 & $b$ & 83.25 & a & 1701.96 & a \\
\hline TMxLP 342 & 0.19 & $f$ & 12.13 & $c$ & 82.88 & a & 1287.96 & $b$ \\
\hline TMxLP 215 & 4.06 & C & 13.25 & $c$ & 82.63 & a & 1358.28 & $b$ \\
\hline TMxLP 220 & 3.10 & c & 42.56 & $a$ & 82.00 & a & 2042.64 & a \\
\hline TMxLP 347 & 2.70 & C & 8.38 & $d$ & 81.63 & a & 1213.08 & $b$ \\
\hline TMxLP 44 & 2.70 & c & 16.88 & $c$ & 80.50 & a & 1403.52 & $b$ \\
\hline TMxLP 71 & 4.06 & $c$ & 13.06 & $c$ & 78.38 & a & 1302.72 & $b$ \\
\hline TMxLP 136 & 3.50 & C & 10.38 & $d$ & 77.13 & a & 1216.68 & $b$ \\
\hline TMXLP 64A & 3.26 & c & 14.50 & $c$ & 76.25 & a & 1302.12 & $b$ \\
\hline TMxLP 149 & 0.26 & $f$ & 21.75 & $b$ & 75.87 & a & 1435.56 & $b$ \\
\hline TMxLP 24 & 0.19 & $f$ & 8.79 & d & 74.41 & a & 1106.16 & b \\
\hline TMxLP 232 & 0.26 & $f$ & 21.25 & $b$ & 74.38 & a & 1405.68 & $b$ \\
\hline TMxLP 336 & 1.50 & $d$ & 10.06 & $d$ & 72.88 & a & 1134.00 & $b$ \\
\hline TMxLP 74 & 0.23 & $f$ & 13.31 & $c$ & 71.75 & a & 1183.20 & $b$ \\
\hline TMxLP 118 & 3.10 & C & 10.38 & d & 71.13 & a & 1139.88 & $b$ \\
\hline TMxLP 325 & 3.50 & c & 23.14 & $b$ & 69.25 & a & 1428.36 & $b$ \\
\hline TMxLP 304 & 1.06 & e & 21.31 & $c$ & 68.87 & a & 1350.60 & $b$ \\
\hline TMxLP 273 & 1.90 & $d$ & 13.56 & $c$ & 68.63 & a & 1171.80 & $b$ \\
\hline TMxLP 69 & 5.57 & c & 48.37 & $a$ & 68.44 & a & 2049.00 & a \\
\hline TMxLP 277 & 0.19 & $f$ & 4.23 & e & 67.75 & a & 916.80 & C \\
\hline TMxLP 66 & 10.62 & $a$ & 20.37 & $b$ & 66.50 & a & 1414.32 & $b$ \\
\hline TMxLP 164 & 0.59 & e & 6.63 & e & 64.75 & a & 943.20 & c \\
\hline TMxLP 205 & 0.66 & e & 10.94 & d & 64.63 & a & 1046.04 & $b$ \\
\hline TMxLP 57 & 0.19 & $f$ & 8.14 & $d$ & 63.50 & a & 959.64 & c \\
\hline TMxLP 34 & 0.99 & e & 5.19 & e & 62.72 & a & 889.08 & c \\
\hline TMxLP 343 & 2.57 & C & 30.07 & $b$ & 61.10 & a & 1485.72 & $b$ \\
\hline TMxLP 228 & 1.46 & $d$ & 26.57 & $b$ & 60.54 & a & 1381.68 & $b$ \\
\hline TMxLP 284 & 2.86 & $c$ & 16.56 & $c$ & 60.12 & a & 1153.20 & $b$ \\
\hline TMxLP 108 & 0.15 & $f$ & 7.10 & e & 59.38 & $b$ & 884.76 & c \\
\hline TMxLP 341 & 1.79 & $d$ & 8.37 & $d$ & 58.19 & $b$ & 920.64 & c \\
\hline TMxLP 46 & 2.66 & c & 14.12 & $c$ & 58.00 & $b$ & 1066.80 & $b$ \\
\hline TMxLP 240 & 0.30 & $f$ & 8.06 & $d$ & 57.88 & $b$ & 891.60 & c \\
\hline TMxLP 280 & 0.19 & $f$ & 4.63 & e & 57.00 & $b$ & 797.40 & c \\
\hline TMxLP 248 & 1.06 & e & 9.50 & $d$ & 56.88 & $b$ & 923.28 & c \\
\hline TMxLP 96 & 1.46 & $d$ & 20.38 & $b$ & 56.25 & $b$ & 1181.64 & $b$ \\
\hline TMxLP 269 & 2.26 & $d$ & 17.38 & $c$ & 55.63 & $b$ & 1111.80 & $b$ \\
\hline TMxLP 30 & 2.22 & $d$ & 7.50 & d & 55.50 & $b$ & 872.64 & c \\
\hline TMxLP 379 & 0.90 & e & 9.40 & d & 55.50 & $b$ & 902.40 & c \\
\hline TMxLP 318 & 2.30 & $d$ & 7.50 & $d$ & 55.38 & $b$ & 872.16 & c \\
\hline TMxLP 47 & 0.70 & e & 6.23 & e & 55.19 & $b$ & 820.20 & c \\
\hline TMxLP 89 & 0.19 & $f$ & 4.79 & e & 54.50 & $b$ & 771.24 & C \\
\hline
\end{tabular}


Continue...

\begin{tabular}{|c|c|c|c|c|c|c|c|}
\hline TMxLP 185 & 0.19 & $f$ & 5.83 & e & 54.31 & $b$ & 793.92 \\
\hline TMxLP 311 & 1.46 & $d$ & 12.44 & c & 54.30 & $b$ & 967.68 \\
\hline TMxLP 112 & 0.19 & $f$ & 2.83 & e & 53.78 & $b$ & 715.56 \\
\hline TMXLP 306 & 0.63 & e & 5.66 & e & 53.25 & $b$ & 782.40 \\
\hline TMXLP 293 & 0.15 & $f$ & 4.39 & e & 52.47 & $b$ & 736.80 \\
\hline TMxLP 365 & 0.23 & $f$ & 4.23 & e & 52.38 & $b$ & 732.84 \\
\hline TMxLP 289 & 0.51 & e & 5.86 & e & 51.12 & $b$ & 760.20 \\
\hline TMxLP 350 & 1.10 & e & 5.19 & e & 50.88 & $b$ & 748.32 \\
\hline TMXLP 180 & 1.10 & e & 5.91 & e & 50.81 & $b$ & 764.76 \\
\hline TMxLP 186 & 0.35 & f & 2.04 & e & 49.75 & $b$ & 650.16 \\
\hline TMxLP 346 & 6.70 & $b$ & 20.50 & $b$ & 49.15 & $b$ & 1162.20 \\
\hline TMXLP 378 & 1.50 & $d$ & 6.06 & e & 49.00 & $b$ & 751.44 \\
\hline TMxLP 287 & 0.11 & f & 7.74 & e & 47.20 & $b$ & 753.48 \\
\hline TMxLP 145 & 0.11 & $f$ & 12.14 & c & 46.79 & $b$ & 854.16 \\
\hline TMxLP 337 & 1.46 & $d$ & 6.06 & e & 46.62 & $b$ & 722.40 \\
\hline TMxLP 167 & 2.62 & d & 8.94 & d & 46.25 & $b$ & 801.00 \\
\hline TMxLP 101 & 1.86 & $d$ & 6.97 & e & 45.06 & $b$ & 730.32 \\
\hline TMxLP 317 & 1.90 & $d$ & 4.06 & e & 44.88 & $b$ & 658.80 \\
\hline TMxLP 39A & 0.22 & f & 8.79 & d & 42.45 & $b$ & 723.00 \\
\hline TMxLP 166 & 0.99 & e & 11.69 & $d$ & 42.41 & $b$ & 801.36 \\
\hline TMXLP 363 & 1.42 & e & 3.02 & e & 41.69 & $b$ & 589.80 \\
\hline TMxLP 370 & 1.46 & d & 8.39 & d & 41.62 & $b$ & 718.32 \\
\hline TMxLP 230 & 2.30 & $d$ & 9.81 & $d$ & 41.37 & $b$ & 759.48 \\
\hline TMxLP 223 & 0.26 & $f$ & 4.06 & e & 40.69 & $c$ & 588.84 \\
\hline TMxLP 241 & 0.59 & e & 6.14 & e & 40.60 & $c$ & 641.64 \\
\hline TMxLP 120 & 1.03 & e & 4.83 & e & 39.19 & $c$ & 598.56 \\
\hline TMxLP 235A & 0.55 & e & 6.70 & e & 38.62 & $c$ & 630.84 \\
\hline TMxLP 332 & 0.19 & $f$ & 20.50 & c & 38.50 & $c$ & 956.28 \\
\hline TMxLP 49 & 2.86 & c & 9.25 & $d$ & 38.37 & $c$ & 716.76 \\
\hline TMXLP 201 & 0.95 & e & 5.54 & e & 38.16 & $c$ & 602.28 \\
\hline TMXLP 373 & 0.91 & e & 6.14 & e & 38.12 & $c$ & 615.72 \\
\hline 'Murcott' & 1.46 & $d$ & 6.30 & e & 37.04 & $c$ & 613.20 \\
\hline TMxLP 331 & 1.42 & e & 6.70 & e & 35.91 & $C$ & 608.76 \\
\hline TMxLP 219 & 0.15 & $f$ & 5.66 & e & 35.81 & $c$ & 567.36 \\
\hline TMxLP 345 & 0.95 & $f$ & 4.70 & e & 35.75 & $c$ & 553.20 \\
\hline TMxLP 177 & 0.19 & $f$ & 8.06 & $d$ & 35.00 & $C$ & 615.72 \\
\hline TMxLP 70 & 1.79 & $d$ & 4.19 & e & 33.91 & $c$ & 528.96 \\
\hline TMxLP 99 & 0.11 & $f$ & 4.84 & e & 33.81 & $c$ & 523.20 \\
\hline TMxLP 236 & 1.50 & $d$ & 9.50 & $d$ & 33.75 & $C$ & 651.00 \\
\hline TMxLP 321 & 0.62 & e & 2.86 & e & 33.72 & $C$ & 480.72 \\
\hline TMxLP 364 & 0.19 & $f$ & 3.50 & e & 32.19 & $c$ & 472.56 \\
\hline TMxLP 250 & 0.59 & e & 5.30 & e & 31.97 & $C$ & 517.92 \\
\hline TMXLP 159 & 1.90 & $d$ & 9.25 & $d$ & 28.50 & $c$ & 586.80 \\
\hline TMxLP 307 & 0.23 & $f$ & 9.19 & $d$ & 28.25 & $c$ & 562.32 \\
\hline TMxLP 281 & 0.66 & e & 5.10 & e & 27.94 & $C$ & 465.60 \\
\hline TMxLP 187 & 0.55 & e & 3.30 & e & 27.56 & $c$ & 416.52 \\
\hline TMxLP 87 & 0.15 & $f$ & 3.79 & e & 27.38 & $c$ & 421.32 \\
\hline TMXLP 295 & 0.15 & $f$ & 8.22 & $d$ & 26.81 & $c$ & 520.80 \\
\hline TMxLP 217 & 1.02 & e & 3.42 & e & 26.79 & $c$ & 415.80 \\
\hline TMxLP 90 & 0.04 & $f$ & 6.88 & $d$ & 25.63 & $c$ & 473.16 \\
\hline TMxLP 303 & 0.95 & e & 8.10 & $d$ & 25.16 & $c$ & 507.72 \\
\hline TMXLP 268 & 0.51 & e & 1.50 & $f$ & 23.75 & $c$ & 327.12 \\
\hline TMxLP 94 & 3.06 & $d$ & 8.35 & e & 22.87 & $d$ & 511.56 \\
\hline TMxLP 247 & 0.95 & e & 2.39 & e & 22.85 & d & 342.96 \\
\hline TMxLP 242 & 0.15 & $f$ & 2.66 & e & 22.72 & d & 338.28 \\
\hline TMxLP 278 & 0.51 & e & 3.42 & e & 22.70 & d & 360.60 \\
\hline TMxLP 261 & 0.55 & e & 0.99 & $f$ & 22.32 & d & 298.20 \\
\hline TMxLP 182 & 0.66 & e & 4.06 & e & 19.94 & d & 344.64 \\
\hline TMXLP 84 & 1.10 & e & 4.06 & e & 19.06 & d & 339.36 \\
\hline TMxLP 152 & 0.15 & f & 6.66 & e & 18.97 & d & 389.28 \\
\hline
\end{tabular}


Continue...

\begin{tabular}{|c|c|c|c|c|c|c|c|}
\hline TMXLP 196 & 0.08 & $f$ & 3.86 & e & 18.91 & d & 320.52 \\
\hline TMxLP 338 & 0.00 & $f$ & 6.06 & e & 18.87 & $d$ & 371.88 \\
\hline TMxLP 395 & 0.63 & e & 4.06 & e & 18.06 & $d$ & 321.72 \\
\hline TMxLP 42 & 0.19 & $f$ & 1.90 & e & 17.41 & $d$ & 256.80 \\
\hline TMxLP 372 & 0.30 & $f$ & 10.69 & $d$ & 17.38 & $d$ & 468.72 \\
\hline TMxLP 176 & 0.08 & $f$ & 1.64 & $f$ & 16.91 & $d$ & 243.24 \\
\hline TMxLP 292 & 0.07 & $f$ & 3.66 & e & 16.44 & $d$ & 285.96 \\
\hline 'Ponkan' & 1.42 & $d$ & 4.26 & e & 16.17 & $d$ & 313.32 \\
\hline TMxLP 207 & 0.08 & $f$ & 2.30 & e & 15.94 & $d$ & 247.44 \\
\hline TMxLP 195 & 1.02 & e & 2.70 & e & 15.82 & $d$ & 266.88 \\
\hline TMxLP 301 & 1.10 & e & 4.14 & e & 14.29 & $d$ & 284.04 \\
\hline TMxLP 227 & 0.15 & $f$ & 7.97 & $d$ & 13.31 & $d$ & 352.80 \\
\hline TMxLP 349 & 0.66 & e & 2.66 & e & 13.19 & $d$ & 230.04 \\
\hline TMxLP 111 & 0.08 & $f$ & 4.14 & e & 12.75 & $d$ & 253.32 \\
\hline TMxLP 351 & 0.15 & $f$ & 6.23 & e & 12.13 & $d$ & 296.88 \\
\hline TMXLP 376 & 0.15 & $f$ & 4.94 & e & 11.88 & $d$ & 262.92 \\
\hline TMxLP 359 & 0.00 & $f$ & 2.22 & e & 11.22 & $d$ & 187.92 \\
\hline TMxLP 334 & 0.07 & f & 1.42 & f & 10.92 & $d$ & 165.96 \\
\hline TMxLP 275 & 0.04 & $f$ & 3.66 & e & 10.69 & $d$ & 216.60 \\
\hline TMxLP 360 & 0.11 & $f$ & 2.30 & e & 9.98 & d & 176.28 \\
\hline TMxLP 116 & 0.48 & e & 0.99 & $f$ & 9.69 & $d$ & 145.80 \\
\hline TMxLP 233 & 0.07 & $f$ & 1.10 & $f$ & 9.65 & $d$ & 143.04 \\
\hline TMxLP 246 & 0.00 & $f$ & 0.19 & $f$ & 9.61 & $d$ & 119.88 \\
\hline TMxLP 315 & 0.59 & e & 1.46 & $f$ & 9.10 & $d$ & 151.32 \\
\hline TMxLP 55 & 0.04 & $f$ & 2.23 & e & 8.85 & $d$ & 160.20 \\
\hline TMxLP 59 & 0.00 & $f$ & 0.00 & $f$ & 7.63 & $d$ & 91.56 \\
\hline TMxLP 60 & 0.00 & $f$ & 4.79 & e & 7.50 & $d$ & 204.96 \\
\hline TMXLP 104 & 0.04 & $f$ & 0.23 & $f$ & 6.79 & $d$ & 87.48 \\
\hline TMxLP 4 & 1.03 & e & 3.26 & e & 5.50 & $d$ & 156.60 \\
\hline TMxLP 291 & 0.15 & $f$ & 1.46 & f & 5.34 & $d$ & 100.92 \\
\hline TMxLP 328 & 0.04 & $f$ & 0.99 & $f$ & 5.34 & $d$ & 88.32 \\
\hline TMxLP 160 & 0.19 & $f$ & 0.66 & $f$ & 4.23 & e & 68.88 \\
\hline TMxLP 200 & 0.00 & $f$ & 1.50 & f & 3.26 & e & 75.12 \\
\hline TMxLP 323 & 0.04 & $f$ & 0.23 & $f$ & 3.10 & e & 43.20 \\
\hline TMxLP 324 & 0.00 & $f$ & 0.00 & $f$ & 2.04 & e & 24.48 \\
\hline TMxLP 260 & 0.00 & $f$ & 0.11 & f & 1.55 & e & 21.24 \\
\hline TMxLP 97 & 0.00 & $f$ & 0.95 & $f$ & 1.50 & e & 40.80 \\
\hline TMxLP 9 & 0.00 & $f$ & 0.00 & $f$ & 1.23 & e & 14.76 \\
\hline TMxLP 221 & 0.08 & $f$ & 0.08 & f & 1.08 & e & 15.84 \\
\hline TMxLP 319 & 0.00 & $f$ & 0.00 & $f$ & 0.19 & e & 2.28 \\
\hline TMxLP 11 & 0.00 & $f$ & 0.04 & $f$ & 0.07 & e & 1.80 \\
\hline TMxLP 5 & 0.00 & $f$ & 0.00 & f & 0.00 & e & 0.00 \\
\hline TMXLP 14 & 0.00 & $f$ & 0.00 & $f$ & 0.00 & e & 0.00 \\
\hline TMxLP 16 & 0.00 & $f$ & 0.00 & $f$ & 0.00 & e & 0.00 \\
\hline TMxLP 18 & 0.00 & $f$ & 0.00 & f & 0.00 & e & 0.00 \\
\hline TMxLP 19 & 0.00 & $f$ & 0.00 & $f$ & 0.00 & e & 0.00 \\
\hline TMxLP 31 & 0.00 & $f$ & 0.00 & $f$ & 0.00 & e & 0.00 \\
\hline TMxLP 40 & 0.00 & $f$ & 0.00 & f & 0.00 & e & 0.00 \\
\hline TMxLP 41 & 0.00 & $f$ & 0.00 & $f$ & 0.00 & e & 0.00 \\
\hline TMxLP 56 & 0.00 & $f$ & 0.00 & $f$ & 0.00 & e & 0.00 \\
\hline TMxLP 75 & 0.00 & f & 0.00 & $\mathrm{f}$ & 0.00 & e & 0.00 \\
\hline TMxLP 88 & 0.00 & $f$ & 0.00 & f & 0.00 & e & 0.00 \\
\hline TMxLP 92 & 0.00 & $f$ & 0.00 & $f$ & 0.00 & e & 0.00 \\
\hline TMxLP 93 & 0.00 & f & 0.00 & f & 0.00 & e & 0.00 \\
\hline TMxLP 107 & 0.00 & $f$ & 0.00 & f & 0.00 & e & 0.00 \\
\hline TMXLP 109 & 0.00 & $f$ & 0.00 & $f$ & 0.00 & e & 0.00 \\
\hline TMxLP 119 & 0.00 & f & 0.00 & f & 0.00 & e & 0.00 \\
\hline TMxLP 146 & 0.00 & f & 0.00 & f & 0.00 & e & 0.00 \\
\hline TMxLP 156 & 0.00 & $f$ & 0.00 & $f$ & 0.00 & e & 0.00 \\
\hline TMxLP 163 & 0.00 & f & 0.00 & f & 0.00 & e & 0.00 \\
\hline TMxLP 169 & 0.00 & 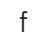 & 0.00 & f & 0.00 & $e$ & 0.00 \\
\hline
\end{tabular}


Continue...

\begin{tabular}{|c|c|c|c|c|c|c|c|c|}
\hline TMXLP 170 & 0.00 & $f$ & 0.00 & $f$ & 0.00 & e & 0.00 & $f$ \\
\hline TMXLP 199 & 0.00 & $f$ & 0.00 & $f$ & 0.00 & e & 0.00 & $f$ \\
\hline TMxLP 202 & 0.00 & $f$ & 0.00 & $f$ & 0.00 & e & 0.00 & $f$ \\
\hline TMxLP 206 & 0.00 & $f$ & 0.00 & $f$ & 0.00 & e & 0.00 & $f$ \\
\hline TMxLP 212 & 0.00 & $f$ & 0.00 & $f$ & 0.00 & e & 0.00 & $f$ \\
\hline TMxLP 213 & 0.00 & $f$ & 0.00 & $f$ & 0.00 & e & 0.00 & $f$ \\
\hline TMxLP 214 & 0.00 & $f$ & 0.00 & $f$ & 0.00 & e & 0.00 & $f$ \\
\hline TMxLP 235 & 0.00 & $f$ & 0.00 & $f$ & 0.00 & e & 0.00 & $f$ \\
\hline TMxLP 245 & 0.00 & $f$ & 0.00 & $f$ & 0.00 & e & 0.00 & $f$ \\
\hline TMxLP 279 & 0.00 & $f$ & 0.00 & $f$ & 0.00 & e & 0.00 & $f$ \\
\hline TMxLP 285 & 0.00 & $f$ & 0.00 & $f$ & 0.00 & e & 0.00 & $f$ \\
\hline TMXLP 288 & 0.00 & $f$ & 0.00 & $f$ & 0.00 & e & 0.00 & $f$ \\
\hline TMxLP 290 & 0.00 & $f$ & 0.00 & $f$ & 0.00 & e & 0.00 & $f$ \\
\hline TMxLP 296 & 0.00 & $f$ & 0.00 & $f$ & 0.00 & e & 0.00 & $f$ \\
\hline TMxLP 299 & 0.00 & $f$ & 0.00 & $f$ & 0.00 & e & 0.00 & $f$ \\
\hline TMXLP 309 & 0.00 & $f$ & 0.00 & $f$ & 0.00 & e & 0.00 & $f$ \\
\hline TMxLP 314 & 0.00 & $f$ & 0.00 & $f$ & 0.00 & e & 0.00 & $f$ \\
\hline TMxLP 327 & 0.00 & $f$ & 0.00 & $f$ & 0.00 & e & 0.00 & $f$ \\
\hline TMxLP 348 & 0.00 & $f$ & 0.00 & $f$ & 0.00 & e & 0.00 & $f$ \\
\hline TMxLP 354 & 0.00 & $f$ & 0.00 & $f$ & 0.00 & e & 0.00 & $f$ \\
\hline TMxLP 362 & 0.00 & $f$ & 0.00 & $f$ & 0.00 & e & 0.00 & $f$ \\
\hline TMXLP 433 & 0.00 & $f$ & 0.00 & $f$ & 0.00 & e & 0.00 & $f$ \\
\hline TMXLP 469 & 0.00 & $f$ & 0.00 & $f$ & 0.00 & e & 0.00 & $f$ \\
\hline TMxLP 887 & 0.00 & $f$ & 0.00 & $f$ & 0.00 & e & 0.00 & $f$ \\
\hline 'Fremont' & 0.00 & $f$ & 0.00 & $f$ & 0.00 & e & 0.00 & $f$ \\
\hline 'Pera' & 0.00 & $f$ & 0.00 & $f$ & 0.00 & e & 0.00 & $f$ \\
\hline $\mathrm{CV}$ & $20 \%$ & & $30 \%$ & & $31 \%$ & & $33 \%$ & \\
\hline
\end{tabular}

to $75.37 \%$. In the last evaluation at 72 hours, 154 hybrids showed symptoms ranging from 0 to $97 \%$ of affected leaf area, corresponding to $75.86 \%$ of the population evaluated in the experiment.

In total, 44 resistant hybrids were observed. Another ten hybrids were tolerant to the disease, since they had low levels of symptoms when compared to other hybrids and did not show statistical differences in relation to asymptomatic plants. It was observed that 86 hybrids presented severity greater than that presented by their susceptible parent, 'Murcott' tangor and nine individuals had severity greater than that of 'Dancy' tangerine, one of the varieties most susceptible to the disease, thus, these materials would not be recommended for areas with high incidence of the disease (Michielin et al., 2016).

TMXLP 281 hybrid, known as the recently launched 'IAC 2019 Maria' tangerine cultivar, stands out. In evaluations under field conditions, it was resistant to ABS; however, it presented mild symptoms (Table 1) in in vitro experiments. The fact that the inoculation was carried out on detached leaves explains in part this phenomenon, since they present physiological changes reflecting on their resistance level; detached leaves lose their power to respond to infections by pathogens (Azevedo et al., 2010; Bastianel et al., 2014).

Studies on the response to inoculation in vivo hybrids are necessary to complement the results, aiming to evaluate the plant's response power to infections, since detached leaves do not have all mechanisms used in plant defense.

The materials used in this study are part of the breeding program of the "Sylvio Moreira" - IAC Citriculture Center. Some hybrids have been evaluated according to their physicochemical characteristics in previous studies, such as that by Michielin et al. (2016) and Curtolo et al. (2017). It was observed that part of the population of hybrids resembled tangors, tangerines and sweet oranges when visual characteristics such as fruit size, ease of peeling, peel thickness, number of segments and seeds were evaluated (Curtolo et al., 2017). Evaluations of soluble solids, ratio and productivity performed by Michielin et al. (2016) complemented the study, and it was possible to verify that some hybrids had agronomic characteristics similar to commercial varieties.

Among the 52 hybrids selected and evaluated by Michielin et al. (2016) 33 of them were also evaluated in the present work, such as TMXLP 14, 16, 47, 107, 111,112 , $116,163,180,233,279,285,324,345,354$ hybrids, which resembled sweet oranges and TMXLP 9, 60, 61, 66, 71, $84,99,118,124,201,281,315,317,321,336,343,370,373$ hybrids, which showed greater similarity to tangors.

There was greater susceptibility in the group of 
tangors when compared to the group of sweet oranges, which presented seven hybrids resistant to ABS (Figure 4). Among hybrids of the tangor group, TMXLP 9, 60, 315 and 84 showed greater tolerance when compared with the others in their group, with emphasis on TMXLP 9, which did not differ statistically from asymptomatic hybrids, presenting potential for being tolerant to $A B S$, with phenotype similar to that of 'Murcott' tangor, currently one of the most cultivated and commercialized species. Tangor fruits are highly appreciated by the Brazilian consumer and excellent option for the domestic and foreign fresh fruit market (Michielin et al., 2016).

\section{A}
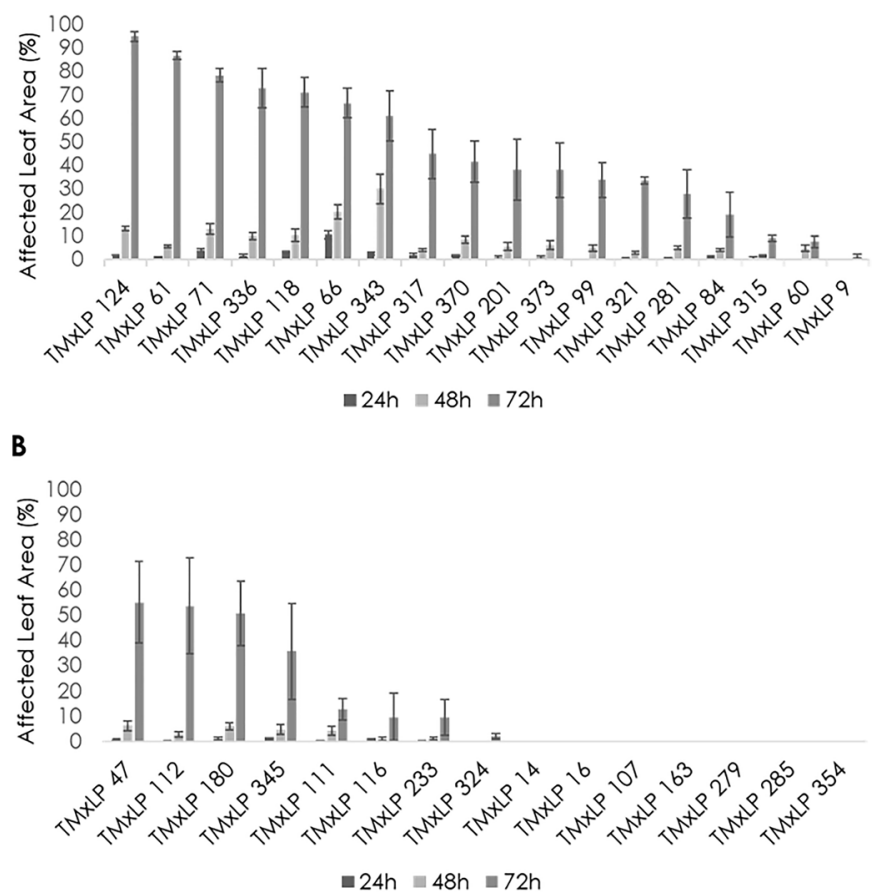

Figure 4. Percentage of leaf area affected by alternaria brown spot after 24, 48 and 72 hours of inoculation in citrus hybrids selected by the Breeding Program of the "Sylvio Moreira / IAC Citriculture Center. A - Group of tangors, B - Group of sweet oranges.

Although 52 hybrids were previously evaluated (Michielin et al., 2016), the present study represents an increase in the volume of collected data, since 198 hybrids were evaluated, supporting future studies on mapping and genomic selection, for example.

Regarding the $198 \mathrm{Fl}$ progenies, it could be concluded that the proportion of susceptible of resistant was 154:44, which apparently corresponds to a 3:1 ratio. The segregation found in this study opposes the hypothesis that the inheritance of $A B S$ resistance is controlled by a single recessive allele, advocated by authors such as Dalkilic et al. (2005); Gulsen et al. (2010); Cuenca et al. (2013) and Michielin et al. (2016).
The origin of the progeny can explain the divergence among segregations found in previous works, since different populations result in different segregations. The hybrids used in this study were obtained through controlled crosses between 'Murcott' Tangor and 'Pera' sweet orange. 'Murcott' tangor is widely known as a natural hybrid of sweet orange and tangerine. Therefore, it can be considered as a backcross, similar to progeny studied by Campos et al. (2017).

However, only population phenotypic data for ABS resistance do not allow identifying the dynamics of alleles involved in the trait. Therefore, genetic and molecular studies are necessary to explain the resistance or susceptibility to A. alternata in citrus.

\section{Conclusions}

Among the 198 hybrids evaluated, 44 are resistant and ten tolerant to A. alternata, demonstrating the greater susceptibility of the population in in vitro assays.

Among the 33 hybrids used in the experiment and which had already been evaluated by the breeding program of the "Sylvio Moreira" Citriculture Center, seven in the group of sweet oranges showed resistance and four in the group of tangors showed tolerance to ABS.

Tolerant hybrids, belonging to the group of tangors, have potential for being used in regions with high incidence of the disease, being an alternative to susceptible varieties.

\section{Acknowledgments}

The authors would like to thank the São Paulo Research Foundation (Fapesp) (processes No. 2016/23155-8, 2019/06412-5, 2018/00133-4, 2016/22133$0,2014 / 50880-0)$ and CNPq (process No. 465440/2014-2) for the support, and to the Laboratory of Phytopathology and Biological Control.

\section{References}

Akimitsu, K., Tsuge, T., Kodama, M., Yamamoto, M., Otani, H. 2014. Alternaria host-selective toxins: determinant factors of plant disease. Journal of General Plant Pathology 80: 109-122.

Azevedo, F.A., Milaneza, T.F., Conceição, P.M., Pacheco, C.A., Martinelli, R., Bastianel, M. 2019. Winter pruning: option for management against alternaria brown spot ('Alteraria alternata' f. sp. 'citri') in Honey Murcott tangor ['Citrus reticulata' Blanco x 'C. sinensis' (L.) Osbeck]. Australian Journal of Crop Science 13: 1631-1337.

Azevedo, F.A, Martelli, I.B., Polydoro, D.A., Pacheco, C.A., Schinor, E.H., Bastianel, M. 2015. Positive relationship between citrus leaf miner and alternaria brown spot. Ciência Rural 45: $1160-1163$. 
Azevedo, F.A., Polydoro, D.A., Bastianel, M., Kupper, K.C., Stuart, R.M., Costa, F.P., Pio, R.M. 2010. Resposta de diferentes genótipos de tangerinas e seus híbridos à inoculação in vitro e in vivo de Alternaria alternata. Revista Brasileira de Fruticultura 32: 944-951.

Barros, J.R.M., Barros, A.L.M., Cypriano, M.P. 2016. O mercado da citricultura no Brasil e suas novas perspectivas. CitrusBR, São Paulo, Brazil. 63 p.

Bastianel, M., Simonetti, L.M., Schinor, E.H., Giorgi Neto, R.O., De Negri, J.D., Gomes, D.N., Azevedo, F.A. 2014. Avaliação do banco de germoplasma de mexericas com relação às características físico-químicas e suscetibilidade à mancha marrom de alternária. Bragantia 73: 23-31.

Campos, K.A., Azevedo, F.A., Bastianel, M., CristofaniYaly, M. 2017. Resistance to alternaria brown spot of new citrus hybrids. Revista Brasileira de Fruticultura 39: e-613.

Canihos, Y., Peever, T.L., Timmer, L.W. 1999. Temperature, leaf wetness, and isolate effects on infection of Minneola tangelo leaves by Alternaria spp. Plant Disease 83: 429433 .

Canteri, M.G., Althaus, R.A., Virgens Filho, J.S., Giglioti, E.A., Godoy, C.V. 2001. SASM-Agri - Sistema para análise e separação de medias em experimentos agrícolas pelos métodos Scott-Knott, Tukey e Duncan. Revista Brasileira de Agrocomputação 1: 18-24.

Cuenca, J., Aleza, P., Vicent, A., Brunel, D., Ollitrault, P. Navarro, L. 2013. Genetically based location from triploid populations and gene ontology of a $3.3-\mathrm{mb}$ genome region linked to alternaria brown spot resistance in citrus reveal clusters of resistance genes. Plos One 8: e76755.

Curtolo, M., Cristofani-Yaly, M., Gazaffi, R., Takita, M.A. Figueira, A., Machado, M.A. 2017. QTL mapping for fruit quality in Citrus using DArTseq markers. BMC Genomics 18: 289.

Dalkilic, Z., Timmer, L.W., Gmitter, F.G. 2005. Linkage of an Alternaria disease resistance gene in mandarin hybrids with RAPD fragments. Journal of the American Society for Horticultural Science 130: 191-195.

Goes, A., Oca, A.G.M, Reis, R.F. 2001. Ocurrencia de la mancha de alternaria em mandarina 'Dancy' en el Estado de Rio de Janeiro. Fitopatologia Brasileira 26: 386.

Gulsen, O., Uzun, A., Canan, I., Seday, U., Canihos, E. 2010. A new citrus linkage map based on SRAP, SSR, ISSR, POGP, RGA and RAPD markers. Euphytica 173: 265- 277.

Huang, F., Fu, Y., Nie, D., Stewart, J.E., Peever T.L., Li, H. 2015. Identification of a novel phylogenetic lineage of Alternaria alternata causing citrus brown spot in China. Fungal Biology 119: 320-330.

IBGE. Instituto Brasileiro de Geografia e Estatística. 2018. Produção Agrícola: Lavoura permanente. https:// cidades.ibge.gov.br/brasil/pesquisa/15/11863<Access on May. 2020>

Martelli, I.B., Pacheco, C.A., Bastianel, M., Schinor, E.H.,
Conceição, P.M., Azevedo, F.A. 2016. Diagrammatic scale for assessing foliar symptoms of alternaria brown spot in citrus. Agronomy Science and Biotechnology 2: 56-61.

Michielin, T.H.V., Cristofani-Yaly, M., Campos, K.A.F., Schinor, E.H., Azevedo, F.A., Bastianel, M. 2016. Reação de híbridos de citros à inoculação com Alternaria alternata. Summa Phytopathologica 42: 313-320.

Oliveira, A.C., Garcia, A.N., Cristofani-Yaly, M., Machado, M.A. 2002. Identification of citrus hybrids through the combination of leaf apex morphology and SSR markers. Euphytica 128: 397-403.

Pacheco, C.A., Martelli, I.B., Polydoro, D.A., Schinor, E.H., Pio, R.M., Kupper, K.C., Azevedo, F.A. 2012. Resistance and susceptibility of mandarins and their hybrids to Alternaria alternata. Scientia Agricola 69: 386-392.

Peever, T.L., Canilhos, Y., Olsen, L., Ibáñez, A., Liu, Y.C., Timmer, L.W. 1999. Population genetic structure and host specificity of Alternaria spp causing brown spot of Minneola tangelo and rough lemon in Florida. Phytopathology 89: 851-860.

Porcino, M.M., Nascimento, L.C., Souza, J.O., Souza, B.O., Nunes, M.C. 2017. Morphogenetic characterization and resistance reaction of mandarin orange genotypes to Alternaria alternata f. sp. citri. Summa Phytopathologica 43: 118-123.

Shaner, G., Finney, R.E. 1977. The effects of nitrogen fertilization on the expression of slow mild wing in knox wheat. Phytopathology 67: 1051-1055.

Silva, A.C., Sales, N.L.P., Araújo, A.V., Caldeira Júnior, C.F. 2009. In vitro effect of plant compounds on the fungus Colletotrichum gloeosporioides Penz. isolated from passion fruit. Ciência e Agrotecnologia 33: 1853-1860.

Soriano, L., Mourão Filho, F.A.A., Carmargo, L.E.A., Cristofani-Yaly, M., Latado, R.R., Pacheco, C.A., Azevedo, F.A., Mendes, B.M.J. 2012. Regeneration and characterization of somatic hybrids combining sweet orange and mandarin/mandarin hybrid cultivars for citrus scion improvement. Plant Cell, Tissue and Organ Culture 111: 385-392.

Sousa, P.F.C.S., Goes, A. 2010. The reaction of sweet oranges as to the resistance of Guignardia citricarpa. Revista Brasileira de Fruticultura 32: 718-725.

Spósito, M.B., Feichtenberger, E., Pio, R.M., Castro, J.L., Renaud, M.S.A. 2003. Ocorrência de mancha marrom de alternaria em diferentes genótipos de citros nos estados de Minas Gerais, São Paulo e Rio Grande do Sul. Fitopatologia Brasileira 28: 231.

Tsuge, T., Harimoto, Y., Akimitsu, K., Ohtani, K., Kodama, M., Akagi, Y. Egusa, M., Yamamoto, M., Otani, H. 2013. Host-selective toxins produced by the plant pathogenic fungus Alternaria alternata. FEMS Microbiology Reviews 37: 44-66.

Turgutoğlu, E., Baktir, I. 2019. In vitro and in vivo assessment of the sensitivity of some tangerine mutants to Alternaria 
alternata pv. citri. Mediterranean Agricultural Sciences 32: $117-120$

Wu, G. et al. 2014. Sequencing of diverse mandarin, pummelo and orange genomes reveals complex history of admixture during citrus domestication. Nature Biotechnology 32: 656-662.

Conflict of Interest Statement: The authors declare that the research was conducted in the absence of any commercial or financial relationships that could be construed as a potential conflict of interest.

All the contents of this journal, except where otherwise noted, is licensed under a Creative Commons Attribution License attribuition-type BY. 